\title{
On Münchhausen numbers
}

\author{
Miroslav Kureš \\ Department of Mathematics, Brno University of Technology \\ Technická 2, 61669 Brno, Czechia \\ e-mail: kures @ fme. vutbr.cz
}

Received: 5 June 2020

Revised: 12 January 2021

Accepted: 24 January 2021

\begin{abstract}
The remarkable property of the number 3435, namely $3435=3^{3}+4^{4}+3^{3}+5^{5}$, was formalized to the notion of Münchhausen numbers. Properties of these numbers are studied and it is showed that although there are only finitely many Münchhausen numbers in a given base $b$, there are infinitely many Münchhausen numbers of the length 2 in all bases. A certain reversion in the definition gives the notion of so-called anti-Münchhausen numbers, search for them is computationally more effective.
\end{abstract}

Keywords: Münchhausen number, Narcissistic number, Sums of powers of integers.

2010 Mathematics Subject Classification: 11A67.

\section{Introduction}

The name of real-life Hieronymus Carl Friedrich von Münchhausen (1720-1797) was anglicized to "Baron Munchausen" and his literary embellished figure is known also under his nicknames, e.g. "Baron Prášil" in Czech (Baron of Lies). We use the original spelling of the family name Münchhausen. There are many stories about this German noblesman, new are still emerging, some of them are even mathematical. One can find the Münchhausen trilemma in logic, the Münchhausen sequence in a coin-weighing puzzle (inspirated by a Russian math Olympiad), etc. In number theory, certain numbers are named Münchhausen numbers. Daan van Berkel wrote in his paper [1]: The reason for picking the name Munchausen number stems from the visual of raising oneself, a feat demonstrated by the famous Baron von Munchausen. Andrew Baxter remarked that the Baron is a narcissistic man indeed, so I think the name is aptly chosen.

The paper is focused on Münchhausen numbers and some of their properties. We will work with different number bases. First, we give a remark about our denotation. For example, for the 
base $b=12$ one can use digits $01234567897 \mathcal{E}$ and the number 131 is expressed as $7 \mathcal{E}$. (Another way is to use letters of the alphabet, which only postpones the need to invent new characters.) We will use decimal numbers, however representing one digit in the base $b$, so $\underbrace{10}_{\mathbf{a}_{1}} \underbrace{11}_{\mathbf{a}_{0}}$. The computing of formula used below is quite easy: $\mathbf{1 0}^{\mathbf{1 0}}+\mathbf{1 1}^{\mathbf{1 1}}=10^{10}+11^{11}=295311670611$ (which is different from 131 and that is why 131 is not the Münchhausen number in the base 12 .

\section{Search for Münchhausen numbers}

Let $\mathbf{a}_{i}, i=0, \ldots, L$ be numbers representing digits of a natural number $n$ in a base $b \in \mathbb{N}-\{1\}$, i.e.,

$$
\sum_{i=0}^{L} \mathbf{a}_{i} b^{i}=n ; \quad \mathbf{a}_{i} \in\{0,1, \ldots, b-1\} .
$$

If

$$
\sum_{i=0}^{L} \mathbf{a}_{i}^{\mathbf{a}_{i}}=n
$$

we call $n$ the Münchhausen number in the base $b$.

It is clear that $1=1^{1}$ is the Münchhausen number for every base $b$; we call it the trivial Münchhausen number. Hereafter, we focus on nontrivial Münchhausen numbers. We note that all $\mathbf{a}_{i}$ must be nonzero as $0^{0}$ is undefined.

Proposition 2.1. Let $n \in \mathbb{N}$ and $\mathbf{a}_{i}$ be as above. If $L \geq b$, then

$$
\sum_{i=0}^{L} \mathbf{a}_{i}^{\mathbf{a}_{i}}<n
$$

holds.

Proof. First, we notice $n \geq b^{L}$ and $(L+1)(b-1)^{b-1}=\sum_{i=0}^{L}(b-1)^{b-1} \geq \sum_{i=0}^{L} \mathbf{a}_{i}^{\mathbf{a}_{i}}$. This means that it suffices to prove

$$
b^{L}>(L+1)(b-1)^{b-1} \quad \text { for all } L>b .
$$

For $L=b$, we have

$$
b^{b}>b^{2}(b-1)^{b-2}>\left(b^{2}-1\right)(b-1)^{b-2}=(b+1)(b-1)^{b-1} .
$$

Let us suppose that the equation is satisfied for $N-1$, i.e.,

$$
b^{N-1}>N(b-1)^{b-1} .
$$

It follows that

$$
b^{N}=b b^{N-1}>b N(b-1)^{b-1}>(N+1)(b-1)^{b-1},
$$

which completes the proof.

Now, although the result is already shown in [1], we come to the following claim in a short and elementary way. 
Proposition 2.2. For every base b, there are finitely many Münchhausen numbers in the base $b$.

Proof. The previous proposition says that there are no Münchhausen numbers for $L \geq b$.

\begin{tabular}{|c|l|}
\hline \hline Base & Nontrivial Münchhausen numbers \\
\hline \hline 3 & 5,8 \\
\hline 4 & 29,55 \\
\hline 6 & 3164,3416 \\
\hline 7 & 3665 \\
\hline 9 & $28,96446,923362$ \\
\hline 10 & 3435 \\
\hline 11 & 34381388,34381640 \\
\hline 12 & 20017650854 \\
\hline 13 & $93367,30033648031,8936504649405,8936504649431$ \\
\hline 14 & $31,93344,17852200903304,606046687989917$ \\
\hline 15 & $614951642128020,11112028004603313,11139050825997348$, \\
\hline 16 & 11139050825997525,11424083732569206 \\
\hline \hline
\end{tabular}

Table 1. All nontrivial Münchhausen numbers in base $b$ from 2 to 16.

Remark 2.1. We say here again that we prefer to consider $0^{0}$ to be undefined. Nevertheless, some people consider $0^{0}=1$ with good reasons, then other numbers come out, see e. g. results of Elizarov in [2]. In our Table 1 such numbers are written by the tiny font. Sometimes it is adopted as even $0^{0}=0$ (sic!), so then the number 438579088 is the second nontrivial Münchhausen number in the base 10 .

\section{Münchhausen numbers of given lengths}

Now, let us focus on Münchhausen numbers of given lengths. A Münchhausen number (in the base b) $n=\sum_{i=0}^{L} \mathbf{a}_{i}^{\mathbf{a}_{i}}$ will be called the Münchhausen number of the length $L+1$. It is evident that the only Münchhausen number of the length 1 is the trivial 1.

There are infinitely many Münchhausen numbers of the length 2 which follows from the following assertion.

Proposition 3.1. A number of the form

$$
\mathbf{a}_{1} \mathbf{a}_{0}=\mathbf{m n}
$$

in a base $b(\mathbf{m}, \mathbf{n} \in\{0,1, \ldots, b-1\})$ is a Münchhausen number of length 2 if and only $m \mid\left(n^{n}-n\right)$ and $b=\frac{n^{n}-n+m^{m}}{m}$. 
Proof. Let $\mathbf{m n}$ be a Münchhausen number in a base $b$. Then

$$
m^{m}+n^{n}=b m+n,
$$

which implies

$$
b=\frac{n^{n}-n+m^{m}}{m} .
$$

As $b=\frac{n^{n}-n}{m}+m^{m-1} \in \mathbb{N}$, we must have $m \mid\left(n^{n}-n\right)$.

The opposite implication is trivial.

In particular, we obtain:

\section{Corollary 3.1.}

(i) Every number of the form $1+n^{n}, n \geq 2$, is the Münchhausen number in the base $1+n^{n}-n$.

(ii) Every number of the form $4+n^{n}, n \geq 2$, is the Münchhausen number in the base $\frac{4+n^{n}-n}{2}$.

Proof. This follows from the previous proposition for the cases $m=1$ and $m=2$. (It is clear that $4+n^{n}-n$ is even for every natural $n$.)

The following claim can be used to recognize when $m$ divides $n^{n}-n$.

Proposition 3.2. Let $p$ be an odd prime number and let $p \mid n$ or $p \mid(n-1)$ or $(p-1) \mid(n-1)$. Then

$$
n^{n}-n \equiv 0 \quad(\bmod p)
$$

Proof. If $p \mid n$, then $n^{n}-n=n\left(n^{n-1}-1\right)$ is easily a multiple of $p$.

If $p \mid(n-1)$, then $n^{n}-n=n\left(n^{n-1}-1\right)$ is also a multiple of $p$, because $n^{n-1}-1$ is a multiple of $n-1$.

Let $(p-1) \mid(n-1)$. As $n^{p-1} \equiv 1(\bmod p)$ (Fermat's Little Theorem), there is also $n^{n-1} \equiv 1(\bmod p)$ which one can $\operatorname{read}$ as $n^{n-1}-1 \equiv 0(\bmod p)$. It follows that $n^{n}-n$ $\equiv 0(\bmod p)$.

The just proven proposition can be used for the following lemma $(p=3)$ as well as for the proposition immediately following the Lemma. Nevertheless, let the elementary proofs be also presented here.

Lemma 3.1. $n^{n}-n$ is divisible by 3 if and only if $n \neq 6 m-4, m \in \mathbb{Z}$.

Proof. We examine all cases $6 m-h, h=0,1, \ldots, 5$. It is clear that $n$ is divisible by 3 for $n=6 m$ and $n=6 m-3$.

Let $n=6 m-1$. Then

$$
(6 m-1)^{6 m-1}-(6 m-1)=\left((6 m)^{6 m-1}-\cdots \text { bin. exp. } \cdots-1\right)-6 m+1
$$

and, of course, $-1+1=0$ and the rest is divisible by 3 . 
Let $n=6 m-5$. Then, for $m=\bar{m}+1$

$$
(6 \bar{m}+1)^{6 \bar{m}+1}-(6 \bar{m}+1)=\left((6 \bar{m})^{6 \bar{m}+1}+\cdots \text { bin. exp. } \cdots+1\right)-6 \bar{m}-1
$$

and, analogously, after the subtraction $1-1=0$ is the rest divisible by 3 .

Let $n=6 m-2$. We have

$$
(6 m-2)^{6 m-2}-(6 m-2)=\left((6 m)^{6 m-2}+\cdots \text { bin. exp. } \cdots+2^{6 m-2}\right)-6 m+2 .
$$

Then, however, it is sufficient to show that $2^{6 m-3}+1$ is divisible by 3 what we prove by induction: for $m=1,9$ is divisible by 3 ; now we derive the validity for $m+1$ under the assumption that the divisibility is satisfied for $m$ by $2^{6 m-3}+1=3 k$. Indeed,

$$
2^{6 m+3}+1=2^{6}\left(2^{6 m-3}+1\right)-2^{6}+1=2^{6} \cdot 3 k-63 .
$$

Let $n=6 m-4$. We have

$$
(6 m-4)^{6 m-4}-(6 m-4)=\left((6 m)^{6 m-4}+\cdots \text { bin. exp. } \cdots+4^{6 m-4}\right)-6 m+4 .
$$

Then, however, it is sufficient to show that $2^{12 m-10}+1$ is non-divisible by 3 what we prove again by induction: for $m=1,5$ is not divisible by 3 ; now we derive the validity for $m+1$ under the assumption that the non-divisibility is satisfied for $m$ by $2^{12 m-10}+1=3 k+\epsilon, \epsilon \in\{-1,1\}$. Indeed,

$$
2^{12 m+2}+1=2^{12}\left(2^{12 m-10}+1\right)-2^{12}+1=2^{12}(3 k+\epsilon)-4095=3\left(2^{12} k-1365\right)+2^{12} \epsilon .
$$

This completes the proof.

Proposition 3.3. Every number of the form $27+n^{n}, n \geq 1, n \neq 6 m-4(m \in \mathbb{N})$ is the Münchausen number in the base $\frac{27+n^{n}-n}{3}$.

Proof. The number $\frac{27+n^{n}-n}{3}$ is an integer if and only if $n^{n}-n$ is divisible by 3 and it was proved that it is satisfied if and only if $n \neq 6 m-4$. We note that $b \geq 2$ already from $n \geq 1$, in fact $b \geq 9$. So, in the base $b=\frac{27+n^{n}-n}{3}, n \geq 1, n \neq 6 m-4$, the expression of $27+n^{n}$ is

$$
\mathbf{a}_{1} \mathbf{a}_{0}=3 \mathbf{n}
$$

Remark 3.1. If we summarize, for every base $b$, there are finitely many Münchhausen numbers in $b$, nevertheless, in all bases, there are infinitely many Münchhausen numbers of length 2 . On the other hand, it seems that there are only finitely many Münchhausen numbers of length $L+1$, where $L \geq 2$. But this interesting conjecture remains unproven. We recommend to look at the following table for eventual considerations in this direction. 


\begin{tabular}{|c|l|c|}
\hline \hline Base & Nontrivial Münchhausen numbers & Length $L+1$ \\
\hline \hline 20 & 50064 & 4 \\
\hline 25 & 3153 & 3 \\
\hline 91 & 49782 & 3 \\
\hline 215 & 46661 & 3 \\
\hline \hline
\end{tabular}

Table 2. Some other nontrivial Münchhausen numbers in various bases.

\section{Anti-Münchhausen numbers}

Let $n, b$ and $\mathbf{a}_{i}, i=0, \ldots, L$ be as above. If

$$
\sum_{i=0}^{L} \mathbf{a}_{i}^{b-\mathbf{a}_{i}}=n
$$

we call $n$ the anti-Münchhausen number in the base $b$.

Evidently, $1=1^{b-1}$ and $b-1=(b-1)^{1}$ are anti-Münchhausen numbers for every base $b$; we call them the trivial anti-Münchhausen numbers. (Let us add also $0=0^{b}$, if we want.)

Hereafter, we focus on nontrivial anti-Münchhausen numbers. We note that $\mathbf{a}_{i}$ can also be zero as $0^{b}$ is well-defined.

\begin{tabular}{|c|l|}
\hline \hline Base & Nontrivial anti-Münchhausen numbers \\
\hline \hline 5 & 17,18 \\
\hline 7 & $65,128,177$ \\
\hline 8 & $256,257,263$ \\
\hline 9 & $178,1123,1993$ \\
\hline 11 & 15524 \\
\hline 13 & 345559 \\
\hline 14 & 883925 \\
\hline 15 & 562452 \\
\hline 16 & 10565939 \\
\hline \hline
\end{tabular}

Table 3. All nontrivial anti-Münchhausen numbers in base $b$ from 2 to 16 .

We find that the upper bound in search for anti-Münchhausen numbers can be taken lower than for Münchhausen numbers. For this, we start with the following lemma.

Lemma 4.1. Let $\mu(b)=\max \left\{\left\lfloor\frac{b}{\mathrm{~W}_{0}(\mathrm{e} b)}\right\rfloor^{b-\left\lfloor\frac{b}{\mathrm{w}_{0}(\mathrm{eb})}\right\rfloor},\left\lceil\left.\frac{b}{\mathrm{~W}_{0}(\mathrm{e} b)}\right|^{b-\left\lceil\frac{b}{\mathrm{w}_{0}(\mathrm{e} b)}\right\rceil}\right\}\right.$, where $\mathrm{W}_{0}(x)$ is the principal value of the Lambert $\mathrm{W}$-function and $\lfloor x\rfloor$ and $\lceil x\rceil$ are usual floor and ceiling functions, respectively. Then $\mu(b)$ is the maximal value of the set $\left\{j^{b-j} ; j=1, \ldots, b-1\right\}$. 
Proof. We compute the maximum of the function $x^{b-x}$. By differentiating, we have to solve

$$
x^{b-x}\left(\frac{b-x}{x}-\log x\right)=0
$$

the solution is

$$
\frac{b}{\mathrm{~W}_{0}(\mathrm{e} b)}
$$

which is somewhere between two consecutive integers. Then

$$
\max \left\{\left\lfloor\frac{b}{\mathrm{~W}_{0}(\mathrm{e} b)}\right\rfloor^{b-\left\lfloor\frac{b}{\mathrm{w}_{0}(\mathrm{e} b)}\right\rfloor},\left\lceil\left.\frac{b}{\mathrm{~W}_{0}(\mathrm{e} b)}\right|^{b-\left\lceil\frac{b}{\mathrm{w}_{0}(\mathrm{e} b)}\right\rceil}\right\}\right.
$$

determines the maximal value of the function $x^{b-x}$ among integral $x$ 's.

Now, we denote by $\nu(b)$ the smallest integer satisfying

$$
(\nu(b)+1) \mu(b)<b^{\nu(b)} .
$$

Some of the values of $\mu(b)$ and $\nu(b)$ are presented in the Table 4. The values of $\nu(b)$ are plotted in the Figure 1. The following proposition summarizes that a computer search for anti-Münchhausen numbers is more effective as to not so high upper bound.

\begin{tabular}{|l|c|c|c|c|c|c|c|c|c|c|c|c|c|c|c|c|}
\hline \hline$b$ & 2 & 3 & 4 & 5 & 6 & 7 & 8 & 9 & 10 & 11 & 12 & 13 & 14 & 15 & 16 & 1000 \\
\hline \hline$\mu(b)$ & 1 & 2 & $2^{2}$ & $3^{2}$ & $3^{3}$ & $3^{4}$ & $2^{8}$ & $2^{10}$ & $2^{12}$ & $2^{14}$ & $5^{7}$ & $5^{8}$ & $5^{9}$ & $2^{9} \cdot 3^{9}$ & $2^{10} \cdot 3^{10}$ & $2^{1672} \cdot 41^{836}$ \\
\hline$\nu(b)$ & 2 & 2 & 2 & 3 & 3 & 3 & 4 & 4 & 5 & 5 & 6 & 6 & 7 & 7 & 8 & 619 \\
\hline \hline
\end{tabular}

Table 4. Numbers $\mu(b)$ and $\nu(b)$ for $b$ from 2 to 16 and for $b=1000$.

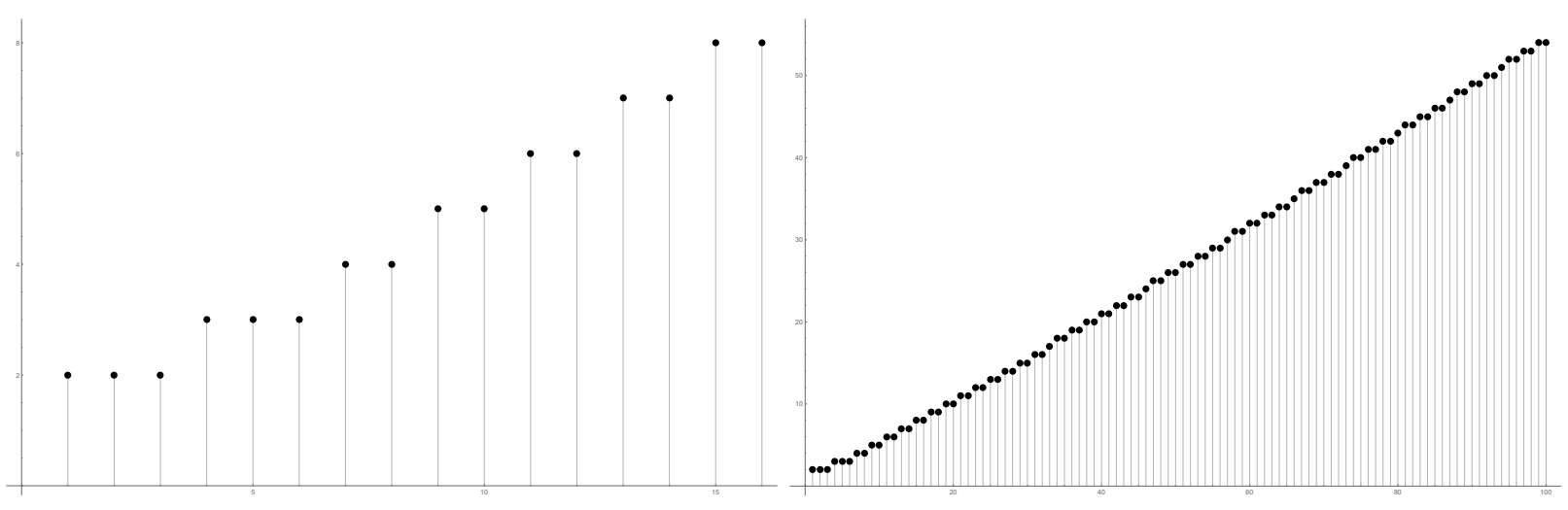

Figure 1. First 16 values (left) and first 100 values (right) of $\nu(b)$.

Proposition 4.1. Let $n \in \mathbb{N}$ and let $\mathbf{a}_{i}, i=0, \ldots, L$ be its digits in a base $b$ and let $L \geq \nu(b)$. Then

$$
n>\sum_{i=0}^{L} \mathbf{a}_{i}^{b-\mathbf{a}_{i}}
$$

holds.

Proof. The proposition follows directly from the definition of $\nu(b)$. 
Remark 4.1. It is also possible to consider an alternative way that, in a certain sense, unifies the approach to upper bounds of Münchhausen numbers and anti-Münchhausen numbers.

As we can adopt $\mu_{j}=\max \left\{j^{b-j} ; j=1, \ldots, b-1\right\}$ as the definition of $\mu_{j}$ in the case of anti-Münchhausen numbers, we can also introduce $\mu_{j}$ in the case of Münchhausen numbers: $\mu_{j}=\max \left\{j^{j} ; j=1, \ldots, b-1\right\}$. Then with conventions already adopted we can compute that $\nu(b)=b$, which gives another proof of Proposition 2.1.

\section{Acknowledgements}

The research has been supported by Brno University of Technology, the specific research plan being No. FSI-S-20-6187. The author would like to thank the unknown reviewer who suggested interesting improvements to this paper.

\section{References}

[1] van Berkel, D., On a curious property of 3435, Available online at: https: / / arxiv . org/pdf/0911.3038v2.pdf

[2] Elizarov, R., MunchausenNumbers/output.txt, Available online at: https://github. com/elizarov/MunchausenNumbers/blob/master/output . txt 